\title{
Perfil Nutricional de Pacientes Onco-Hematológicos Internados em um Hospital Especializado em Câncer em São Luís - MA
}

\author{
Nutritional Profile of Onco-hematological Patients Hospitalized in a Cancer \\ Specialized Hospital in São Luís - MA
}

\author{
Rafaela Lopes Holanda ${ }^{1}$ \\ Eliete Costa Oliveira ${ }^{2}$ \\ Gilberth Silva Nunes ${ }^{3}$ \\ Carlos Eduardo Pires Galvão ${ }^{4}$ \\ Igor Nunes Rego e Silva ${ }^{5}$ \\ Gabriela Sander de Sousa Nunes ${ }^{6}$
}

\section{RESUMO}

Objetivo: Avaliar o perfil nutricional de pacientes onco-hematológicos internados em um hospital especializado em câncer em São Luís - MA. Metodologia: Estudo transversal, retrospectivo, analítico, com coleta de dados secundária, envolvendo pacientes com idade mínima de 18 anos, de ambos os gêneros e que tenham sido submetidos a pelo menos uma ASG-PPP (Avaliação Subjetiva Global Produzida Pelo Paciente). Os dados foram coletados em registros do Serviço de Nutrição e Dietética do hospital. Analisaram-se dados demográficos (gênero e idade), clínicos (diagnóstico) e nutricionais: Índice de massa corporal (IMC), circunferência braquial (CB), prega cutânea tricipital (PCT), circunferência muscular do braço (CMB) e ASG-PPP. As análises foram realizadas no programa estatístico Stata ${ }^{\circledR}$ 13.0. O nível de significância utilizado para os testes foi de $p<0,05$. Resultados: Foram avaliados 330 pacientes, onde foi constatado que $67,58 \%$ eram de adultos e $32,42 \%$ de idosos, com maior frequência do sexo masculino, com $60,30 \%$. Ocorreu maior incidência de leucemia (58,48\%), seguidos de linfomas (24,85\%), mieloma múltiplo $(13,33 \%)$ e síndrome mielodisplásica (3,3\%). Na avaliação do estado nutricional os resultados mostraram que a ASG-PPP detectou maior número de pacientes com algum grau de desnutrição do que outros indicadores (93,94\%), seguido pela PCT $(65,76 \%)$, CMB $(53,64 \%)$, CB $(45,45 \%)$ e IMC $(14,87 \%)$. De acordo com o IMC, foi encontrado maior incidência de eutrofia, correspondendo a $57,27 \%$ da amostra. Conclusão: Diante do que foi encontrado, destaca-se que a desnutrição é um aspecto de extrema importância a ser considerado no tratamento de pacientes onco-hematológicos, visto que pode interferir diretamente no prognóstico da doença.

DESCRITORES

Perfil Nutricional. Desnutrição. Onco-Hematologia.

\begin{abstract}
Objective: To evaluate the nutritional profile of onco-hematological patients admitted to a specialized cancer hospital in São Luís - MA. Methodology: Cross-sectional, retrospective, analytical study, with secondary data collection, involving patients aged at least 18 years, of both genders and having undergone at least one ASG-PPP (Subjective Global Assessment Produced by the Patient). Data were collected from records of the Hospital's Nutrition and Dietetics Service. Demographic (gender and age), clinical (diagnostic) and nutritional: Body Mass Index (BMI), Brachial Circumference (CB), Tricipital Skinfold (PCT), Muscular Arm Circumference (CMB) and ASG-PPP data were analyzed. The analyses were performed using the Stata ${ }^{\circledR} 13.0$ statistical program. The level of significance used for the tests was $p<0.05$. Results: 330 patients were evaluated, in which it was found that $67.58 \%$ are adults and $32.42 \%$ are elderly, with a higher frequency of males with $60.30 \%$. There was a higher incidence of Leukemia $(58.48 \%)$, followed by Lymphomas $(24.85 \%)$, Multiple Myeloma (13.33\%) and Myelodysplastic Syndrome (3.3\%). In the assessment of nutritional status, the results showed that ASG-PPP detected a greater number of patients with some degree of malnutrition than other indicators $(93.94 \%)$, followed by PCT $(65.76 \%)$, CMB (53.64\%), CB (45.45\%) and BMI (14.87\%). According to the BMI a higher incidence of eutrophy was found, which corresponds to $57.27 \%$ of the sample. Conclusion: In view of what was found, it is highlighted that malnutrition is an extremely important aspect to be considered in the treatment of onco-hematological patients, since it can directly interfere in the prognosis of the disease.
\end{abstract}

DESCRIPTORS

Nutritional Profile. Malnutrition. Onco-Hematology.

${ }^{1}$ Pós-Graduanda em Residência Multiprofissional em Saúde em Atenção em Oncologia pela Secretaria de Estado da Saúde do Maranhão - SES/MA. Nutricionista Residente do Hospital de Câncer do Maranhão Tarquínio Lopes Filho - HCMATLF, São Luis-MA - Brasil. ${ }^{2}$ Mestre em Saúde Materno-Infantil pela Universidade Federal do Maranhão-UFMA. Nutricionista do Hospital da Criança / Hospital Universitário do, São Luis-MA - Brasil.

${ }^{3}$ Mestre em Saúde e Ambiente pela Universidade Federal do Maranhão-UFMA. Docente do Curso de Nutrição das Faculdades Estácio São Luis, UNINASSAU - São Luis e UNDB - São Luis, São Luis-MA - Brasil.

${ }^{4}$ Pós-Graduado em Residência Multiprofissional em Saúde em Terapia Intensiva pela Universidade Federal do Maranhão - UFMA. Nutricionista do Hospital Dr. Carlos Macieira e Preceptor de estágio da Faculdade Pitágoras, São Luis-MA - Brasil.

${ }^{5}$ Pós-Graduado em Nutrição Clínica e Funcional pela Faculdade Estácio de São Luis-MA. Nutricionista do Hospital de Câncer do Maranhão Tarquínio Lopes Filho - HCMATLF, São Luis-MA - Brasil.

${ }^{6}$ Pós-Graduada em Nutrição Clínica e Funcional pela Faculdade Estácio de São Luis-MA. Nutricionista do Hospital de Câncer do Maranhão Tarquínio Lopes Filho - HCMATLF, Preceptora da Residência Multiprofissional em Saúde em Atenção em Oncologia e Terapia Intensiva da Secretaria de Estado da Saúde - SES/MA, São Luis-MA - Brasil. 
câncer é considerado um importante problema de saúde pública no Brasil e no mundo como a doença de maior impacto socioeconômico entre todas as causas de morte ${ }^{1}$. Estima-se um crescimento das taxas globais de $50 \%$ entre os anos de 2000 e 2020, o que deve resultar em uma incidência de 10 a 15 milhões de pacientes com câncer².

O número de novos casos de câncer cresce a cada ano. Para o Brasil, a estimativa do Instituto Nacional de Câncer José Alencar Gomes da Silva ${ }^{3}$ para cada ano do triênio 2020-2022 aponta que ocorrerão 625 mil casos novos de câncer (450 mil, excluindo os casos de câncer de pele não melanoma). O câncer de pele não melanoma será o mais incidente (177 mil), seguido pelos cânceres de mama e próstata (66 mil cada), cólon e reto (41 mil), pulmão (30 mil) e estômago (21 mil).

A etiologia da doença é multifatorial, podendo ser causada por fatores internos (mutações hereditárias, hormonais, condições imunológicas e mutações resultantes do metabolismo) ou fatores externos (tabaco, químicos, organismos infecciosos e radiação). Estes fatores causais podem agir em conjunto ou em sequência para iniciar ou promover o desenvolvimento do câncer4 .

Em virtude disso, é interessante destacar as neoplasias malignas hematológicas, que compreendem principalmente as leucemias, linfomas e mielomas. Estas são caracterizadas por alterações no sistema imunológico que, em geral, são resultantes de uma combinação de fatores determinantes da própria doença, bem como do tratamento antineoplásico. A rigor, todos os componentes básicos da defesa do organismo podem ser afetados: a pele, as mucosas, a imunidade celular específica e inespecífica e a imunidade humoral, em particular, a síntese hepática da proteína de fase aguda ${ }^{5}$.

O câncer hematológico tem relevância pública pelo fato da descoberta repentina do diagnóstico e da necessidade de internação imediata, sendo mais prevalente as leucemias (originadas da medula óssea) e os linfomas (originadas de qualquer outro órgão linfoide) ${ }^{6}$.

Os pacientes com doença hematológica têm um risco aumentado de desnutrição, em que a incidência da perda de peso no diagnóstico consiste em 30 a $40 \%$ nos linfomas de Hodgkin e das leucemias não linfocítica aguda e 50 a $60 \%$ em formas mais agressivas de linfomas não Hodgkin?

A perda de peso é tida como fator de risco nutricional primário em indivíduos com câncer, sendo consequente ao hipercatabolismo da própria doença e ao tratamento quimioterápico que favorece o aparecimento de complicações de impacto nutricional, tornando-se necessárias intervenções dietéticas adequadas $^{8}$.

Diante disso, pacientes oncológicos comumente apresentam alterações fisiológicas (hipermetabolismo/hipercatabolismo), sintomas gastrointestinais e ingestão dietética insuficiente, associados à própria doença e ao tratamento ${ }^{9}$. A avaliação nutricional objetiva identificar o risco de desnutrição ou de deficiência de nutrientes nos pacientes, quantificar o risco de complicações clínicas ligadas à desnutrição e controlar a adequação da terapia nutricional ${ }^{10}$.

Logo, para avaliar o estado nutricional de pacientes oncológicos, métodos rápidos, de fácil aplicabilidade e de baixo custo são utilizados no intuito de identificar o risco nutri- 
cional ou a desnutrição, além de intervir com a terapêutica nutricional o mais precocemente possível ${ }^{11}$.

Os métodos antropométricos atualmente disponíveis para avaliar a condição nutricional e a inadequação quase absoluta de qualquer método ou ferramenta, se usados isoladamente, demonstrando a ausência de um padrão-ouro. Assim, diferentes métodos foram combinados, em uma tentativa de aumentar a especificidade e a sensibilidade da avaliação nutricional ${ }^{12}$.

O uso da Avaliação subjetiva global produzida pelo paciente (ASGPPP) em combinação com um protocolo que inclua um tratamento oncológico, pode triar os pacientes que venham a se beneficiar de uma intervenção específica. Torna-se um bom método na prática clínica por incluir dados descritivos relativos à perda de peso, mudanças na ingestão alimentar, impacto de sintomas gastrointestinais, capacidade funcional, exame físico, dentre outros ${ }^{13}$.

Nesse sentido, o suporte nutricional é essencial em indivíduos com diagnóstico de câncer, uma vez que a desnutrição tem impacto negativo sobre a evolução da doença e a continuidade terapêutica ${ }^{14}$. Dessa forma a identificação e o manejo precoce de problemas nutricionais podem melhorar o prognóstico dos pacientes oncológicos, reduzindo deficiências nutricionais.

Em virtude da existência de poucos estudos que avaliaram o estado nutricional em pacientes com câncer hematológico, o principal objetivo deste estudo foi avaliar o perfil nutricional de pacientes onco - hematológicos internados em um hospital especializado em câncer em São Luís - MA.

\section{METODOLOGIA}

Trata-se de um estudo transversal, retrospectivo, analítico, com coleta de dados secundária, realizada entre os meses de março e abril de 2019, com pacientes internados entre setembro de 2014 a outubro de 2018, no Hospital de Câncer do Maranhão Tarquínio Lopes Filho - HCMATLF, em São Luís, Maranhão, Nordeste do Brasil.

A população do estudo correspondeu ao total de pacientes arquivados no sistema eletrônico do Serviço de Nutrição e Dietética do hospital, totalizando 330 pacientes. Foram selecionados pacientes com idade mínima de 18 anos, de ambos os gêneros, com neoplasias onco-hematológicas (leucemia, linfoma, mieloma múltiplo e síndrome mielodisplásica) e terem sido submetidos, pelo menos, a uma ASG-PPP. Foram excluídos pacientes com dados incompletos.

O estado nutricional foi avaliado por cinco métodos distintos: ASG-PPP: variável categorizada em: A (bem nutrido), B (moderadamente desnutrido ou suspeito de desnutrição) ou C (gravemente desnutrido).

Foi utilizada a ASG-PPP na versão traduzida e validada por Gonzalez et al. ${ }^{15}$. A parte inicial do formulário contém perguntas sobre mudanças recentes no peso corporal, realização de atividades cotidianas, alterações na ingestão alimentar e sintomas que a influenciam e é preenchida com informações geradas pelo participante da pesquisa. $\mathrm{O}$ restante do questionário foi respondido pelos nutricionistas assistenciais, com informações sobre o diagnóstico do paciente, o estresse metabólico e o exame físico.

O índice de massa corporal (IMC): va- 
riável contínua obtida pela razão do peso em quilos e o quadrado da altura em metros ${ }^{16}$. A variável foi classificada para adultos e idosos conforme padrões estabelecidos pela $\mathrm{WHO}^{16}$ e por Lipschitz ${ }^{17}$ para adultos e idosos.

A circunferência do braço (CB): variável contínua, o resultado foi obtido pela medida da CB será comparado aos valores de referência de NHANES III (National Health Nutrition Examination Survey), demonstrado na tabela de percentis por Frisancho ${ }^{18}$. Primeiramente, foi calculado o percentual de adequação da $\mathrm{CB}$, de acordo com a fórmula: $\%$ adequação de $\mathrm{CB}=\mathrm{CB}$ obtida $\div \mathrm{CB}$ percentil $50 \times 100$.

A prega cutânea tricipital (PCT): variável contínua, o resultado foi obtido pela medida da PCT e foi comparado com os valores de referência de NHANES III (National Health Nutrition Examination Survey), demonstrado na tabela de percentis por Frisancho ${ }^{18}$. Primeiramente, foi calculado o percentual de adequação da PCT de acordo com a fórmula: $\%$ adequação de $\mathrm{PCT}=\mathrm{PCT}$ obtida $\div$ PCT percentil $50 \times 100$.

A circunferência muscular do braço (CMB): variável contínua, a partir das medidas de CB e PCT será calculada a CMB, aplicando-se a seguinte fórmula: $\mathrm{CMB}(\mathrm{cm})=$ CB $(\mathrm{cm})-\pi \times$ [PCT $(\mathrm{mm}) / 10]$, onde $\pi=3,14$. $\mathrm{O}$ resultado obtido pelo cálculo da $\mathrm{CMB}$ foi comparado com os valores de referência de NHANES III (National Health Nutrition Examination Survey), demonstrado na tabela de percentis por Frisancho ${ }^{19}$. Primeiramente, foi calculado o percentual de adequação da CMB de acordo com a fórmula: \% adequação de $\mathrm{CMB}=\mathrm{CMB}$ obtida $\div \mathrm{CMB}$ percentil $50 \times 100$.

Os dados foram tabulados e analisa- dos no programa estatístico Stata ${ }^{\circledR}$ 13.0. As variáveis qualitativas foram apresentadas em valores absolutos e relativos. Para a comparação entre as proporções foi aplicado o teste do qui-quadrado de Pearson. O nível de significância utilizado para os testes foi de p<0,05.

O presente estudo foi aprovado pelo Comitê de Ética em Pesquisa com Seres Humanos do Hospital Universitário da Universidade Federal do Maranhão (UFMA), sob o Protocolo $n^{\circ}$ 3.393.135, de 14 de junho de 2019. A pesquisa seguiu as orientações fornecidas na Resolução CNS n¹96, de 10 de outubro de 1996, do Conselho Nacional de Saúde.

\section{RESULTADOS}

Foram analisados 330 pacientes por meio do sistema de Nutrição e Dietética do HCMATLF, onde constatou-se que $67,58 \%$ $(n=223)$ são de adultos e $32,42 \%(n=107)$ são de idosos, com maior frequência do sexo masculino, com 60,30\% ( $n=199)$. Caracterizando a amostra quanto aos tipos de cânceres onco-hematológicos mais prevalentes, observou-se que 58,48\% ( $n=193)$ foram de leucemias, $13,33 \%(n=44)$ foram de linfomas, $24,85 \%$ ( $n=82$ ) foram de mieloma múltiplo e $3,3 \%(n=11)$ foram de síndrome mielodisplásica (Tabela 1).

O estado nutricional avaliado pela ASG-PPP mostrou que $62,73 \%(n=207)$ dos pacientes foram classificados com desnutrição moderada. De acordo com o IMC, foi encontrada maior incidência de eutrofia, que corresponde a $57,27 \%(n=189)$ da amostra. Por outro lado, segundo a classificação da $\mathrm{CB}, \mathrm{PCT}$ e CMB, a maior parte dos pacientes 
Tabela 1. Frequência de sexo, idade e diagnóstico de pacientes onco-hematológicos internados em São Luís, 2019.

\begin{tabular}{lll}
\hline Variáveis & $\mathrm{n}$ & $\%$ \\
\hline Sexo & 199 & 60,30 \\
$\quad$ Masculino & 131 & 39,70 \\
$\quad$ Feminino & & \\
Idade & 223 & 67,58 \\
$\quad$ Adulto & 107 & 32,42 \\
$\quad$ Idoso & & \\
Diagnóstico & 193 & 58,48 \\
$\quad$ Leucemia & 44 & 13,33 \\
Mieloma Múltiplo & 82 & 24,85 \\
$\quad$ Linfoma & 11 & 3,33 \\
$\quad$ Síndrome mielodisplásica & 330 & 100 \\
\hline Total & & \\
\hline
\end{tabular}

Tabela 2. Frequência da avaliação do estado nutricional de pacientes onco-hematológicos internados em São Luís, 2019.

\begin{tabular}{lll}
\hline Variáveis & $\mathrm{n}$ & $\%$ \\
\hline ASG PPP & 20 & 6,06 \\
Bem nutrido & 207 & 62,73 \\
Desnutrido moderado & 103 & 31,21 \\
Desnutrido grave & & \\
IMC & 68 & 20,61 \\
Desnutrido & 189 & 57,27 \\
Eutrofia & 73 & 22,12 \\
Sobrepeso/Obesidade & & \\
CB & 150 & 45,45 \\
Desnutrido & 130 & 39,39 \\
Eutrofia & 50 & 15,15 \\
Sobrepeso/Obesidade & & \\
PCT & 217 & 65,76 \\
Desnutrido & 58 & 17,58 \\
Eutrofia & 55 & 16,67 \\
Sobrepeso/Obesidade & & \\
CMB & 177 & 53,64 \\
Desnutrido & 153 & 46,36 \\
Eutrofia & 330 & 100 \\
\hline Total &
\end{tabular}

IMC: Índice de Massa Corporal; CB: Circunferência do Braço; PCT: Prega Cutânea Tricipital; CMB: Circunferência Muscular do Braço; ASG-PPP: Avaliação Subjetiva Global Produzida Pelo Paciente. 
Tabela 3. Diagnóstico nutricional segundo Índice de Massa Corporal, Circunferência do Braço, Prega Cutânea Tricipital e Circunferência Muscular do Braço, em pacientes oncohematológicos internados em São Luís, 2019.

\begin{tabular}{|c|c|c|c|c|c|}
\hline Idade IMC & $\begin{array}{l}\text { Desnutrido } \\
\mathrm{n}(\%)\end{array}$ & $\begin{array}{l}\text { Eutrófico } \\
\mathrm{n}(\%)\end{array}$ & $\begin{array}{l}\text { Sobrep./Obeso } \\
\mathrm{n}(\%)\end{array}$ & $\begin{array}{l}\text { Total } \\
\mathrm{n}(\%)\end{array}$ & $p$ value \\
\hline Adulto & $28(12,56)$ & $140(62,78)$ & $55(24,66)$ & $223(67,58)$ & 0,00 \\
\hline Idoso & $40(37,38)$ & $49(45,80)$ & $18(16,82)$ & $107(32,42)$ & \\
\hline Total & $68(20,61)$ & $189(57,27)$ & $73(22,12)$ & $330(100,0)$ & \\
\hline $\begin{array}{ll}\text { Sexo } & \text { CB } \\
\end{array}$ & $\begin{array}{l}\text { Desnutrido } \\
\mathrm{n}(\%)\end{array}$ & $\begin{array}{l}\text { Eutrófico } \\
\mathrm{n}(\%)\end{array}$ & $\begin{array}{l}\text { Sobrep./Obeso } \\
\mathrm{N}(\%)\end{array}$ & $\begin{array}{l}\text { Total } \\
\text { n (\%) }\end{array}$ & $p$ value \\
\hline Masculino & $82(41,21)$ & $74(37,19)$ & $43(21,60)$ & $199(60,30)$ & 0,000 \\
\hline Feminino & $68(51,91)$ & $56(42,75)$ & $07(5,34)$ & $131(39,70)$ & \\
\hline Total & $150(45,45)$ & $130(39,40)$ & $50(15,15)$ & $330(100,0)$ & \\
\hline $\begin{array}{ll}\text { Idade } & \text { CB } \\
\end{array}$ & $\begin{array}{l}\text { Desnutrido } \\
\mathrm{n}(\%)\end{array}$ & $\begin{array}{l}\text { Eutrófico } \\
\mathrm{n}(\%)\end{array}$ & $\begin{array}{l}\text { Sobrep./Obeso } \\
\mathrm{n}(\%)\end{array}$ & $\begin{array}{l}\text { Total } \\
\mathrm{n}(\%)\end{array}$ & $p$ value \\
\hline Adulto & $124(55,60)$ & $77(34,53)$ & $22(9,87)$ & $223(67,58)$ & 0,000 \\
\hline Idoso & $26(37,38)$ & $53(45,80)$ & $28(16,82)$ & $107(32,42)$ & \\
\hline Total & $150(45,45)$ & $130(39,40)$ & $50(15,15)$ & $330(100,0)$ & \\
\hline Sexo PCT & $\begin{array}{l}\text { Desnutrido } \\
\mathrm{n}(\%)\end{array}$ & $\begin{array}{l}\text { Eutrófico } \\
\mathrm{n}(\%)\end{array}$ & $\begin{array}{l}\text { Sobrep./Obeso } \\
\mathrm{n}(\%)\end{array}$ & $\begin{array}{l}\text { Total } \\
\mathrm{n}(\%)\end{array}$ & $p$ value \\
\hline Masculino & $120(60,30)$ & $35(17,59)$ & $44(22,11)$ & $199(60,30)$ & 0,004 \\
\hline Feminino & $97(74,04)$ & $23(17,56)$ & $11(8,40)$ & $131(39,70)$ & \\
\hline Total & $217(65,76)$ & $58(17,57)$ & $55(16,67)$ & $330(100,0)$ & \\
\hline Idade & $\begin{array}{l}\text { Desnutrido } \\
\mathrm{n}(\%)\end{array}$ & $\begin{array}{c}\text { Eutrófico } \\
\mathrm{n}(\%)\end{array}$ & $\begin{array}{l}\text { Sobrep./Obeso } \\
\mathrm{n}(\%)\end{array}$ & $\begin{array}{c}\text { Total } \\
\mathrm{n}(\%) \\
\end{array}$ & $p$ value \\
\hline Adulto & $136(60,99)$ & $45(20,18)$ & $42(18,83)$ & $223(67,58)$ & 0,031 \\
\hline Idoso & $81(75,70)$ & $13(12,15)$ & $13(12,15)$ & $107(32,42)$ & \\
\hline Total & $217(65,76)$ & $58(17,57)$ & $55(16,67)$ & $330(100,0)$ & \\
\hline Sexo $\quad$ CMB & $\begin{array}{l}\text { Desnutrição } \\
\mathrm{n}(\%)\end{array}$ & $\begin{array}{l}\text { Eutrofia } \\
\mathrm{n}(\%)\end{array}$ & $\begin{array}{l}\text { Total } \\
\mathrm{n}(\%) \\
\end{array}$ & & $p$ value \\
\hline Masculino & $134(67,34)$ & $65(32,66)$ & $199(60,30)$ & & 0,000 \\
\hline Feminino & $43(32,82)$ & $88(67,18)$ & $131(39,70)$ & & \\
\hline Total & $177(53,64)$ & $153(46,36)$ & $330(100,0)$ & & \\
\hline $\begin{array}{ll}\text { Idade } & \text { CMB } \\
\end{array}$ & $\begin{array}{l}\text { Desnutrido } \\
\mathrm{n}(\%)\end{array}$ & $\begin{array}{c}\text { Eutrofia } \\
\mathrm{n}(\%)\end{array}$ & $\begin{array}{c}\text { Total } \\
\mathrm{n}(\%)\end{array}$ & & $p$ value \\
\hline Adulto & $129(57,85)$ & $94(42,15)$ & $223(67,58)$ & & 0,027 \\
\hline Idoso & $48(44,86)$ & $59(55,14)$ & $107(32,42)$ & & \\
\hline Total & $177(53,64)$ & $153(46,36)$ & $330(100,0)$ & & \\
\hline $\begin{array}{ll}\text { CMB } & \text { CMnost. } \\
\end{array}$ & $\begin{array}{l}\text { Desnutrido } \\
\mathrm{n}(\%)\end{array}$ & $\begin{array}{l}\text { Eutrofia } \\
\mathrm{n}(\%)\end{array}$ & $\begin{array}{l}\text { Total } \\
\mathrm{n}(\%)\end{array}$ & & $p$ value \\
\hline Leucemia & $108(55,96)$ & $85(44,04)$ & $193(58,48)$ & & 0,002 \\
\hline Mieloma & $21(47,73)$ & $23(52,27)$ & $44(13,33)$ & & \\
\hline Linfoma & $48(58,54)$ & $34(41,46)$ & $82(24,85)$ & & \\
\hline SM & - & $11(100,0)$ & $11(3,33)$ & & \\
\hline Total & $177(53,64)$ & $153(46,36)$ & $330(100,0)$ & & \\
\hline
\end{tabular}


Tabela 4. Diagnóstico nutricional segundo Avaliação Subjetiva Global Produzida Pelo Paciente, em pacientes onco-hematológicos internados em São Luís, 2019.

\begin{tabular}{|c|c|c|c|c|c|}
\hline ASGPPP & $\begin{array}{c}\text { Bem Nutrido } \\
\text { n (\%) }\end{array}$ & $\begin{array}{c}\text { Desn. Moderada } \\
\mathrm{n}(\%)\end{array}$ & $\begin{array}{c}\text { Desn. Grave } \\
\mathrm{n}(\%)\end{array}$ & $\begin{array}{l}\text { Total } \\
\mathrm{n}(\%)\end{array}$ & $p$ value \\
\hline Masculino & $12(6,03)$ & $138(69,35)$ & $49(24,62)$ & $199(60,30)$ & 0,005 \\
\hline Feminino & $08(6,11)$ & $69(52,67)$ & $54(41,22)$ & $131(39,70)$ & \\
\hline Total & $20(6,06)$ & $207(62,73)$ & $103(31,21)$ & $330(100,0)$ & \\
\hline ASGPPP & $\begin{array}{l}\text { Bem Nutrido } \\
\mathrm{n}(\%)\end{array}$ & $\begin{array}{l}\text { Desn. Moderada } \\
\mathrm{n}(\%)\end{array}$ & $\begin{array}{l}\text { Desn. Grave } \\
\text { n (\%) }\end{array}$ & $\begin{array}{l}\text { Total } \\
\text { n (\%) }\end{array}$ & $p$ value \\
\hline Adulto & $15(6,72)$ & $130(58,30)$ & $78(34,98)$ & $223(67,58)$ & 0,056 \\
\hline Idoso & $05(4,68)$ & $77(71,96)$ & $25(23,36)$ & $107(32,42)$ & \\
\hline Total & $20(6,06)$ & $207(62,73)$ & $103(31,21)$ & $330(100,0)$ & \\
\hline
\end{tabular}

Teste: qui-quadrado de Pearson; $\mathrm{P}<0,05$

ASGPPP: Avaliação Subjetiva Global produzida Pelo Paciente.

Tabela 5. Perfil demográfico de pacientes onco-hematológicos segundo diagnóstico clínico em São Luís, 2019.

\begin{tabular}{|c|c|c|c|c|c|c|}
\hline Sexo Diagn. & $\begin{array}{l}\text { Leucemia } \\
\mathrm{n}(\%)\end{array}$ & $\begin{array}{l}\text { Mieloma } \\
\mathrm{n}(\%)\end{array}$ & $\begin{array}{l}\text { Linfoma } \\
\mathrm{n}(\%)\end{array}$ & $\begin{array}{l}\mathrm{SM} \\
\mathrm{n}(\%)\end{array}$ & $\begin{array}{l}\text { Total } \\
\mathrm{n}(\%)\end{array}$ & $p$ value \\
\hline Masculino & $114(57,29)$ & $29(14,57)$ & $48(24,12)$ & $08(4,02)$ & $199(60,30)$ & 0,678 \\
\hline Feminino & $79(60,30)$ & $15(11,45)$ & $34(25,95)$ & $03(2,30)$ & $131(39,70)$ & \\
\hline Total & $193(58,48)$ & $44(13,33)$ & $82(24,85)$ & $11(3,33)$ & $330(100,0)$ & \\
\hline Idade Diagn. & $\begin{array}{l}\text { Leucemia } \\
\mathrm{n}(\%)\end{array}$ & $\begin{array}{c}\text { Mieloma } \\
\mathrm{n}(\%)\end{array}$ & $\begin{array}{l}\text { Linfoma } \\
\mathrm{n}(\%)\end{array}$ & $\begin{array}{c}\text { SM } \\
n(\%)\end{array}$ & $\begin{array}{l}\text { Total } \\
\text { n (\%) }\end{array}$ & $\mathrm{P}$ value \\
\hline Adulto & $139(62,33)$ & $22(9,87)$ & $60(26,90)$ & $02(0,90)$ & $223(67,58)$ & 0,000 \\
\hline Idoso & $54(50,47)$ & $22(20,56)$ & $22(20,56)$ & $09(8,41)$ & $107(32,42)$ & \\
\hline Total & $193(58,48)$ & $44(13,33)$ & $82(24,85)$ & $11(3,33)$ & $330(100,0)$ & \\
\hline
\end{tabular}

Teste: qui-quadrado de Pearson; $\mathrm{P}<0,05$

SM: Síndrome Mielodisplásica.

encontravam-se desnutridos, com $45,45 \%$ $(n=150), 65,76 \%(n=217)$ e $53,64 \%(n=177)$, respectivamente (Tabela 2 ).

Ao analisar o diagnóstico nutricional segundo o IMC, da CB, da PCT e da CMB em pacientes onco-hematológicos, verificou-se que em todas as comparações efetuadas en- tre o IMC com a variável idade, a CB e a PCT com as variáveis sexo e idade e a $\mathrm{CMB}$ com as variáveis sexo, idade e diagnóstico, ocorreu relação significativa $(p<0,05)$ (Tabela 3$)$.

No que se refere ao diagnóstico nutricional, segundo a ASG-PPP, em pacientes onco-hematológicos, observou-se que ao 
equiparar as variáveis sexo e a idade, existiu uma relação significativa com a variável sexo ( $p<0,05)$, no entanto, não ocorreu significância ao comparar com a variável idade $(p=0,056)$ (Tabela 4).

Com relação ao perfil demográfico de pacientes onco-hematológicos segundo diagnóstico clínico, considerou-se que existiu uma relação significativa ao compará-lo com a variável idade $(p<0,05)$, todavia, não ocorreu significância ao acarear a variável sexo $(p=0,678)($ Tabela 5).

\section{DISCUSSÃO}

A desnutrição é uma complicação frequente em pacientes oncológicos sendo a avaliação nutricional precoce de extrema importância para minimizar os riscos de complicações e melhorar o estado nutricional ${ }^{20}$.

A prevalência da desnutrição em pacientes com neoplasias hematológicas é pouco estabelecida na literatura. Neste caso, muitas vezes tendo um grande impacto, uma vez que pode piorar ou prolongar a imunossupressão induzida pelo tratamento antineoplásico, aumentando o risco de complicações infecciosas, sendo a principal causa de morbidade e mortalidade durante o tratamento ${ }^{21}$.

No presente estudo, observou-se que o tipo de câncer onco-hematológico mais prevalente foi a leucemia, totalizando $58,48 \%$ da amostra, sendo $62,33 \%$ pacientes adultos (Tabela 5). Em outro estudo com 127 pacientes, onde foi avaliado o risco nutricional de pacientes onco-hematológicos, pode-se averiguar que $74,1 \%$ eram de pacientes diagnosticados com leucemia, sendo $72,7 \%$ pacientes adultos ${ }^{22}$.

De acordo com dados do INCA, a estimativa de casos novos para homens e mulheres no estado do Maranhão e na capital São Luís para o ano de 2020, serão de 270 e 60 casos de leucemia, respectivamente, superior a de outros dados de cânceres hematológicos ${ }^{9}$.

Poucos estudos avaliaram o estado nutricional em cânceres hematológicos no Brasil. Analisando a avaliação antropométrica nesse estudo, identificou-se uma prevalência de $57,27 \%$ de eutrofia, de acordo com o IMC, sendo $62,78 \%$ pacientes adultos (Tabela 3 ).

Sabe-se que o IMC possui um valor limitado em pacientes oncológicos, uma vez que estes pacientes podem apresentar aumento dos parâmetros inflamatórios, como citocinas, o que pode acarretar degradação proteica e a expansão de líquido extracelular, ocasionando retenção hídrica, edema e mascarando o estado nutricional. Ainda, em muitas formulações de quimioterapia utilizam glicorticóides e/ou terapia hormonal, os quais também provocam retenção hídrica, degradação da massa muscular e edema, mascarando o peso atual ${ }^{23}$.

Sendo a avaliação nutricional capaz de predizer o desfecho clínico e o prognóstico do paciente, estudos têm demonstrado que avaliar isoladamente algum indicador nutricional pode gerar falhas no diagnóstico nutricional ${ }^{24}$. Por outro lado, ao analisar outros parâmetros antropométricos ( $\mathrm{CB}, \mathrm{PCT}$ e $\mathrm{CMB}$ ) nesse estudo, verificou-se que a maior parte dos pacientes encontra-se desnutridos (Tabela 3 ).

Esses dados corroboram com outros estudos, no qual Thieme et $\mathrm{al}^{24}$ observou que a maior prevalência de pacientes desnutridos foi vista pela PCT, seguida de $C B$, da $C M B$, da ASG-PPP e do IMC, em um estudo com 125 pacientes, onde encontrou-se maior percentual de desnutridos graves pela PCT $(31,2 \%)$, quando comparado com a ASG-PPP, a CMB e 
o IMC. O IMC, um dos índices mais utilizados na prática clínica, se mostra um método frágil que pode subestimar a desnutrição ${ }^{25}$.

Isto configura um agravo à saúde do paciente oncológico, pois o déficit nutricional é capaz de reduzir a resposta terapêutica, transformar sua autoimagem, predispor a maiores riscos infecciosos e elevar a morbimortalidade, reduzindo, assim, sua qualidade de vida ${ }^{26}$.

O estado nutricional dos pacientes onco-hematológicos, avaliados por meio da ASG-PPP, constatou que $93,94 \%$ dos pacientes apresentaram algum grau de desnutrição, sendo a maioria adultos $(n=208)$ e do sexo masculino $(n=187)$ (Tabela 4).

De acordo com um estudo realizado no Serviço de Hematologia do Instituto Nacional de Cancerologia do México, na realização da ASG-PPP em pacientes onco-hematológicos, observou-se que $50,4 \%$ dos pacientes tinham algum grau de desnutrição, sendo que $31,1 \%$ apresentavam desnutrição moderada, enquanto, $19,3 \%$ dos pacientes apresentavam desnutrição grave ${ }^{27}$.

Em outro estudo avaliando doentes onco-hematológicos, encontrou-se uma prevalência de $60,5 \%$ de pacientes com algum grau de desnutrição na admissão. Além disso, pacientes desnutridos apresentaram maior taxa de readmissões, o que mostra a importância da triagem e da avaliação nutricional em pacientes com câncer, a fim de proporcionar intervenção nutricional precoce ${ }^{28}$.

Cabe ressaltar, que no Brasil a incidência de desnutrição em pacientes oncológicos, foi abordada no estudo multicêntrico - Inquérito Brasileiro de Avaliação Nutricional - IBRANUTRI, o qual demonstrou que os pacientes oncológicos internados têm ocorrência de desnutrição quase três vezes maior do que os demais sem a doença, incluindo o câncer como fator de risco para a mesma. Neste mesmo estudo, $66,3 \%$ dos pacientes com câncer apresentaram algum grau de desnutrição pela ASG-PPP ${ }^{29}$.

Uma das limitações desse estudo foi encontrar trabalhos para confrontar com os resultados encontrados. Além disso, foi necessário comparar a população dessa pesquisa com públicos de diferentes diagnósticos oncológicos devido à escassez de trabalhos que fizessem uma relação de pacientes onco-hematológicos com indicadores antropométricos.

\section{CONCLUSÃO}

Diante do que foi encontrado, destaca-se que a desnutrição é um aspecto de extrema importância a ser considerado no tratamento de pacientes onco-hematológicos, visto que pode interferir diretamente no prognóstico da doença.

Com isso, propõe-se que maior atenção seja destinada ao estado nutricional desses pacientes, por meio da realização de uma avaliação nutricional completa, pois, esta é capaz de identificar deficiências nutricionais que podem culminar no agravamento do quadro clínico. Essas alterações possuem relevância tamanha, tal que a realização de uma antropometria incompleta, baseada somente no IMC, poderia ser omitido a desnutrição aqui evidenciada pela CB, PCT, CMB e ASG-PPP.

Assim, preza-se pelo estudo dos vários aspectos preditivos da nutrição do enfermo, ressaltando-se que a análise do estado nutricional do paciente onco-hematológico é uma ferramenta de apoio essencial ao tratamento.

Novos estudos devem ser realizados visando estabelecer uma intervenção nutricional precoce com objetivo de melhorar a recuperação e proporcionar uma melhor qualidade de vida nestes pacientes. 


\section{REFERÊNCIAS}

1. Navarro, AM, Japur, CC, Sicchieri, JMF. Atualidades em Alimentação e Nutrição Hospitalar. 1.ed. Rio de Janeiro: Atheneu, 2017.

2. Ferlay, J. et al. Estimated cancer incidence and mortality world wide in 2012. IARC Cancer Base n. 11. International Agency for Research Cancer, Lyon, 2013.

3. Brasil. Ministério da Saúde. Secretaria de Atenção à Saúde Instituto Nacional de Câncer José Alencar Gomes da Silva. Brasil terá 625 mil novos casos de câncer a cada ano do triênio 2020-2022. [Internet]. Brasília: Ministério da Saúde, 2020

4. Pascoal, TSF. Intervenção nutricional em oncologia. Porto, 2012.

5. Burigo, T, Fagundes, RLM, Trindade, EBSM, Vasconcelos, HCFF, Massaut, IH B, Rotolo, MAS. Ação do prebiótico sobre as proteínas de fase aguda de pacientes com neoplasia hematológica. Rev. Bras. Hematol. Hemoter. 2007; 29(2):130-135.

6. Hashisaka, DF. Terapia Ocupacional em Hematologia e Transplante de Medula Óssea: uma revisão integrativa de literatura. Ribeirão Preto. 2017

7. Laviano, A, Meguid, MM, Inui, A, Muscaritoli, M, Rossi-Fanelli, F. Therapy insight: Cancer anorexia-cachexia syndrome: when all you can eat is yourself. Nat Clin Pract Oncol.2005; 2(3):158-165.

8. Baracos, VE. Body composition in patients with non-small cel llung cancer: a contemporary view of cancer cachexia with the use of computed tomography image analysis. Am J Clin Nutr. 2010; 91(4):1133-1137.

9. Brasil. Ministério da Saúde. Secretaria de Atenção à Saúde Instituto Nacional de Câncer José Alencar Gomes da Silva. Inquérito Brasileiro de Nutrição Oncólogica. [Internet]. Brasília: Ministério da Saúde, 2013.

10. Poziomyck, AK. Avaliação nutricional pré-operatória e risco cirúrgico em pacientes com tumores do trato gastrintestinal superior. [Internet]. Porto Alegre: Universidade Federal do Rio Grande do Sul, 2011.

11.Silva, MPN. Síndrome da anorexia-caquexia em portadores de câncer. Revista Brasileira de Cancerologia. 2006; 52(1):59-77.

12.Ryu, SW, Kim, IH. Comparison of different nutritional assessments in detecting malnutrition among gastric cancer patients. World J Gastroenterol.2010; 16(26):3310-3317.

13.Santos, AF. Avaliação subjetiva global produzida pelo paciente e sobrevivência: estudo em hospital de referência em câncer de São Luís (MA). (Dissertação de Mestrado). São Luís: Universidade Federal do Maranhão; 2014

14.Chasen, M, Ashbury, F. Nutrition as supportive care in the cancer experience. Support Care Cancer.2010;18 (Supl. 02):11-12.

15. Gonzalez, MC, Borges, LR, Silveira, DH, Assunção, MCF, Orlandi, SP Validação da versão em português da avaliação subjetiva global produzida pelo paciente. Rev Bras de Nutrição Clínica. 2010; 25(2):102-108.

16. World Health Organization (WHO). Physical status: the use and interpretation of anthropometric. Geneva: WHO; 1995.

17. Lipschitz, DA. Screening for nutritional status in theelderly. Prim Care.1994; 21(1):55-67.
18. Frisancho, AR. Anthropometric standards :an interactive nutritional reference of body size and body composition for children and adults. 2 . ed. University of Michigan; 2008.

19. Frisancho, AR. New norms of upper limb fat and muscle areas for assessment of nutritional status. Am J Clinical Nutrition, 1981 34(11):2540-2545

20. Chagas, CL, Silva, CP. Análise comparativa entre métodos de avaliação nutricional em pacientes oncológicos. Revista De Trabalhos Acadêmicos Universo Recife. 2017; 4(2):1-14

21. Gómez-Candela, $\mathrm{C}$ et al. Nutritional intervention in oncohematological patient. Nutr Hosp. 2012; 27(3):669-80.

22. Rodrigues, BC, Sales, AEC, Rodrigues, BC, Mendonça, OS, Aguiar APN, Daltro, AFCS. Rev Bras Cancerologia. 2019; 65(1):1-7.

9. Brasil. Ministério da Saúde. Secretaria de Atenção à Saúde Instituto Nacional de Câncer José Alencar Gomes da Silva. Inquérito Brasileiro de Nutrição Oncólogica. [Internet]. Brasília: Ministério da Saúde, 2013.

23. Tartari, RF, Busnello, FM, Nunes, CHA. Perfil Nutricional de Pacientes em Tratamento Quimioterápico em um Ambulatório Especializado em Quimioterapia. Rev Bras Cancerologia, 2010; 56(1):43-50.

24. Thieme, RD. Cutchma, G, Chieferdecker, MEM, Campos, CL. O Índice De Risco Nutricional (Nutritional Risk Index) É Preditor De Complicação Pós-Operatória Em Operações Do Aparelho Digestivo Ou Parede Abdominal? ABCD Arq Bras Cir Dig, 2013; 26(4):286-292.

25. Santos, CAD. Estado Nutricional e Fatores Associados À Desnutrição em Idosos em Tratamento Oncológico. (Dissertação mestrado online) Viçosa: Universidade Federal de Viçosa,2013.

26. Brito, LF et al. Perfil Nutricional de Pacientes com Câncer Assistidos pela Casa de Acolhimento ao Paciente Oncológico do Sudoeste da Bahia. Rev Bras Cancerologia, 2012; 58(2):163-171.

27. Luna, E. Baltazar, Guzmán, L. I. Omaña, Hernández, L. Ortiz, Ñamendis-Silva, S. A, Delfin, L. De Nicola. Estado nutricioen pacientes de primer ingreso a hospitalización del Servicio de Hematología de Instituto Nacional de Cancerología. Madrid, 2013.

28. Calleja, FA. et al. Food in take and nutritional status influence out comes in hospitalized hematology-oncology patients. Nutr Hosp. 2015; 31(6):2598-2605

29. Waitzberg DL, Caiaffa WT, Correia MI. Hospital malnutrition: the Brazilian national survey (IBRANUTRI): a study of 4000 patients. Nutrition. $2001 ; 17(7-8): 573-580$

CORRESPONDÊNCIA

Rafaela Lopes Holanda

Rua de Santaninha, 178 - Centro

São Luis - MA - Brasil - CEP 65010-580

E-mail: rafaelanutri@gmail.com 